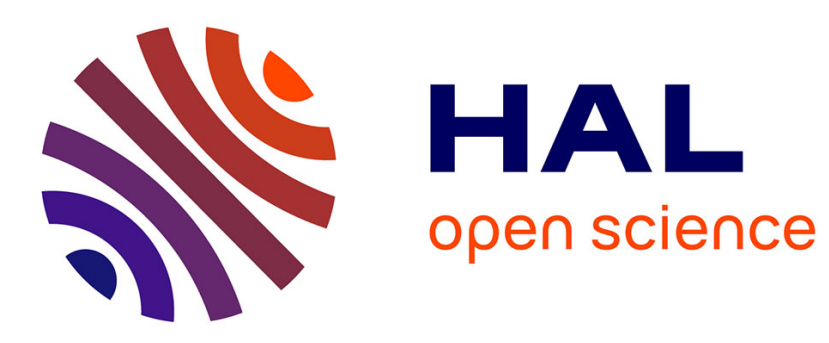

\title{
Adaptive Leader-Follower Formation in Cluttered Environment Using Dynamic Target Reconfiguration
}

\author{
Jose Miguel Vilca Ventura, Lounis Adouane, Youcef Mezouar
}

\section{To cite this version:}

Jose Miguel Vilca Ventura, Lounis Adouane, Youcef Mezouar. Adaptive Leader-Follower Formation in Cluttered Environment Using Dynamic Target Reconfiguration. 12th International Symposium on Distributed Autonomous Robotic Systems (DARS 2014), Nov 2014, Daejeon, South Korea. pp.237254, 10.1007/978-4-431-55879-8_17. hal-01712911

\section{HAL Id: hal-01712911 \\ https://hal.science/hal-01712911}

Submitted on 23 Feb 2018

HAL is a multi-disciplinary open access archive for the deposit and dissemination of scientific research documents, whether they are published or not. The documents may come from teaching and research institutions in France or abroad, or from public or private research centers.
L'archive ouverte pluridisciplinaire HAL, est destinée au dépôt et à la diffusion de documents scientifiques de niveau recherche, publiés ou non, émanant des établissements d'enseignement et de recherche français ou étrangers, des laboratoires publics ou privés. 


\title{
Adaptive Leader-Follower Formation in Cluttered Environment using Dynamic Target Reconfiguration
}

José Vilca, Lounis Adouane and Youcef Mezouar

\begin{abstract}
This paper presents a control architecture for safe and smooth navigation of a group of Unmanned Ground Vehicles (UGV) while keeping a specific formation. The formation control is based on Leader-follower and Behavioral approaches. The proposed control architecture is designed to allow the use of a single control law for different multi-vehicle contexts (navigation in formation, transition between different formation shapes, obstacle avoidance, etc.). The obstacle avoidance strategy is based on the limit-cycle approach while taking into account the dimension of the formation. A new Strategy for Formation Reconfiguration (SFR) of the group of UGVs based on suitable smooth switching of the set-points (according, for instance, to the encountered obstacles or the new task to achieve) is proposed. The inter-vehicles collisions are avoided during the SFR using a penalty function acting on the vehicle velocities. Different simulations on cluttered environments show the performance and the efficiency of the proposal, to obtain fully reactive and distributed control strategy for the navigation in formation of a group of UGVs.
\end{abstract}

\section{Introduction}

In the last decades, research interest in control and coordination of multiple robots has increased significantly. Different tasks that may be performed by a single complex robot can be performed with more flexibility and efficiency by a group of elementary cooperative robots. These cooperatives robots join their capacities and information to improve the task achievement.

Exploration [16], management and platooning of autonomous vehicles [5], mapping of unknown locations [19], coverage of unknown area [9], transport of heavy objects [3], rendez-vous of multiple agents [21] are some examples of multi-robot tasks.

J. Vilca, L. Adouane and Y. Mezouar

Institut Pascal, Blaise Pascal University - UMR CNRS 6602, Clermont-Ferrand, France

e-mail: FirstName.Lastname@univ-bpclermont.fr

This work was supported by the French National Research Agency through the Safeplatoon project. 
Fig. 1 Autonomous navigation in formation of a group of UGVs in an urban environment (Clermont-Ferrand, France).

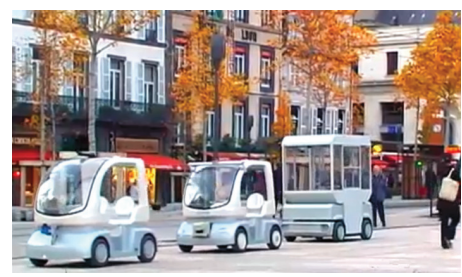

Nonetheless, the coordination of multi-robot navigation is among the most challenging tasks, due notably, to its implication for instance for public transportation [15]. This paper addresses the navigation of a group of vehicles in formation (i.e., when a group of mobile robots has to navigate and to keep a desired relative positions to each other or to a reference). Mostly, three approaches have been investigated to deal with this problem: behavior-based [22], virtual structure [10] and Leader-follower [12] approaches. In this work, the proposed control architecture is based on Leader-follower and behavior-based approaches for the formation control problem.

In the Leader-follower approach, the leader is the reference for the desired configuration of the followers. Different works exploit graph theory to describe the inter-vehicle communications [5, 8, 18, 20]. Different formation cases (leader reassignment, robot adding and control saturation) were presented in [18]. The authors proposed a formation control law based on the combination of Linear Matrix Inequalities and hybrid system. The case of dynamic formation, i.e., the formation shape changes to another (e.g. from square to triangle), and obstacle avoidance was dealt in $[5,8,20]$. In [5], the leader generates a free-collision trajectory in a dynamic environment which is tracked using a formation control law based on neural network, Lyapunov function and dynamic model of the robot. The stability of the dynamic formation and dynamic topology (adjacency matrix) are also demonstrated. In [8], switches between different formation shapes are exploited (from triangle to line) to avoid encountered obstacles in the environment. The formation control law is based on input-output feedback linearization and vision sensors (omnidirectional camera) are embedded in each robot for localization and navigation purpose. A strategy to modify the formation configuration by scaling the distance between the vehicles is proposed in [20]. Obstacle avoidance is dealt with using potential fields. In the already presented works, interesting solutions for formation control problem are proposed. Nevertheless, they are based on predefined trajectories and do not address the issues related to the constraints of the formation shape and to the dimension of the vehicles (large vehicles need large space for navigation and obstacle avoidance). In this paper, the main proposal is to present a fully reactive and distributed navigation in formation of a fleet of UGVs. Additionally, we make a focus on the reconfiguration phase based on a suitable smooth switches between the formation shapes while considering the follower configurations.

In this paper, the dynamics of the followers' set-points are given by the specific dynamic of the leader (Leader-follower approach). This strategy allows a good flexibility proprieties for the formation shape [7]. The behavior-based approach allows to use different elementary controllers to perform several sub-tasks (cf. Fig. 2). The 
obstacle avoidance controller for the whole formation is based on limit-cycle principle [1]. This control architecture is designed in order that each follower tracks safely the assigned configuration (given by the leader) while using an appropriate dynamic target-reaching controller. The proposed strategy for formation reconfiguration takes into account the presence of obstacles in the environment as well as the inter-vehicles distance to avoid collision between the UGVs.

The rest of this paper is organized as follows: in the next section, the control architecture for the navigation of UGVs in formation is introduced. The model of the UGV and its controllers are also detailed. In Section 3, the navigation in formation based on Leader-follower approach is described. Moreover, this section presents also the reconfiguration method for the multi-robot formation. Simulations showing the efficiency of the proposed strategy are detailed in Section 4. Finally, conclusion and future works are given in Section 5.

\section{Control architecture}

The control architecture for the navigation in formation of a group of UGVs is shown in Fig. 2. This control architecture is designed for a group of UGVs modeled as tricycle robots. This architecture aims to manage the interactions among elementary controllers while guaranteeing the stability of the overall control [2]. It allows to obtain safe and smooth navigation of the formation (cf. section 3). The global navigation in formation framework is operated by the Formation parameters block that sends to each elementary controller (Dynamic target reaching and Obstacle avoidance) its desired set-points. Each elementary controller (cf. Fig. 2) provides as output a Control Input $\mathbf{C I}$ to the Control law block through a Hierarchical action selection block which selects between $\mathbf{C I}_{T}$ or $\mathbf{C I}_{O}$.

In this work, a single control law for the UGV (tricycle robot) is used. It considers the vehicle poses and velocities. This control law allows the UGV to reach a static or dynamic target with a desired orientation and velocity (cf. subsection 2.2.3). The inputs of the control law (pose errors between the vehicle and its assigned target) are provided by the elementary controllers (cf. subsection 2.2). The control law is synthesized according to Lyapunov theorem (more details are given in [23]). The main blocks of the architecture are detailed below.

The Perceptions and communication block incorporates the propriocetive and exteroceptive sensors such as range sensor, cameras, odometers and RTK-GPS. Its goal is to capture information related to the robot environment, mainly potential obstacles $[6,8]$. The communication is related with the vehicle capability to send and to receive information from other vehicles. In the sequel, we assume a stable communication between UGVs without latency) and that each UGV has a RTKGPS and a range sensor LIDAR.

The Formation parameters block determines the desired configuration for the group of UGVs to keep a specific distance and orientation between them. The Leader-follower approach is used to obtain the formation configuration of the UGVs 
according to the Leader configuration (cf. Section 3). Moreover, a reconfiguration strategy between the formation shapes according to the context of the environment (dynamic and/or cluttered or not) is described in subsection 3.2.

The control architecture uses a Hierarchical action selection mechanism to manage the switches between the two elementary controllers (Behavior-based approach), Dynamic target reaching and Obstacle avoidance blocks, according to the formation parameters and environment perception. The hierarchical action selection mechanism activates the Obstacle avoidance block as soon as it detects at least one obstacle which can hinder the future vehicle movement toward its dynamic virtual target (more details are given in [1]). It allows to anticipate the activation of obstacle avoidance controller and to decrease the time to reach the assigned target (static or dynamic). In order to provide the enough overall details of the presented control architecture, the following subsections present the UGV model and elementary controllers.

\subsection{Vehicle and target set-point modeling}

We assume that the UGVs evolves in asphalt road and in cluttered urban environment with relatively low speed (less than $v_{\max }=2 \mathrm{~m} / \mathrm{s}$ ). Hence, the use of kinematic model (which relies on pure rolling without slipping) of the UGV is sufficient. The kinematic model of the UGV is based on the well-known tricycle model [17]. The two front wheels are replaced by a single virtual wheel located at the center between the front wheels. The equations of UGV model can be written as (cf. Fig. 3):

$$
\dot{x}=v \cos (\theta) ; \quad \dot{y}=v \sin (\theta) ; \quad \dot{\theta}=v \tan (\gamma) / l_{b}
$$

where $(x, y, \theta)$ is the $\mathrm{UGV}$ pose in the global reference frame $X_{G} Y_{G} \cdot v$ and $\gamma$ are respectively the linear velocity and the orientation of the vehicle front wheel. $l_{b}$ is the wheelbase of the vehicle.

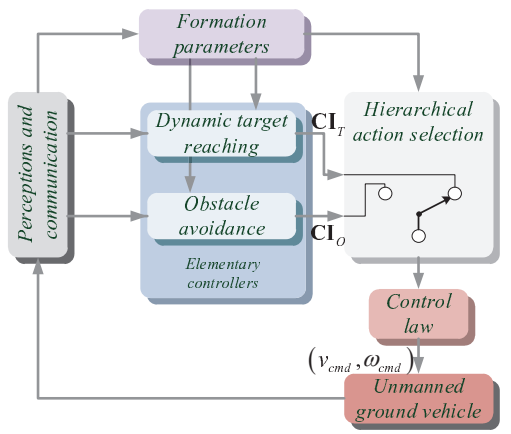

Fig. 2 Proposed control architecture embedded in each UGV navigating in the formation.

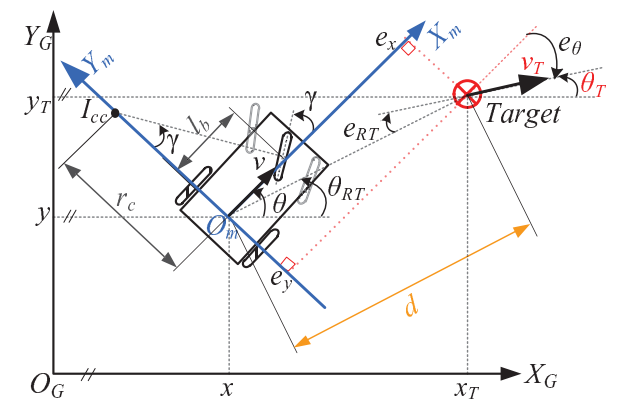

Fig. 3 UGV and dynamic target configuration variables in (local and global) Cartesian reference frames. 


\subsection{Elementary controllers}

Each elementary controller generates the control inputs $\mathbf{C I}$ (pose errors $\left(e_{x}, e_{y}, e_{\theta}\right)$ and velocities $v_{T}$ ) of the Control law block (cf. Fig. 2).

\subsubsection{Dynamic target reaching controller}

The target set-point modeling is defined by the formation shape (Formation parameters block) and it is computed according to the leader configuration (cf. Section 3 ). The target is defined by $\left(x_{T}, y_{T}, \theta_{T}\right)$ and $v_{T}$ which are respectively the target poses and linear velocity in the global reference frame. Indeed, an RTK-GPS embedded in each vehicle allows to estimate its current configuration.

Before to introduce the control law, let us describe the following notations (cf. Fig. 3):

- $I_{c c}$ is the instantaneous center of curvature of the vehicle trajectory, $r_{c}=l_{b} / \tan (\gamma)$ is the radius of curvature and $c_{c}=1 / r_{c}$ is the curvature.

- $\left(e_{x}, e_{y}, e_{\theta}\right)$ are the errors w.r.t local frame $\left(X_{m} Y_{m}\right)$ between the vehicle and the target poses.

- $\theta_{R T}$ and $d$ are respectively the angle and distance between the target and vehicle positions.

- $e_{R T}$ is the error related to the vehicle position $(x, y)$ w.r.t the target orientation.

This controller guides the vehicle towards the dynamic target. It is based on the pose control of the UGV w.r.t. the target (represented by errors variables $\left(e_{x}, e_{y}, e_{\theta}\right)$ in Fig. 3). These errors are computed w.r.t the local reference frame $X_{m} Y_{m}$ and they are given by:

$$
\left\{\begin{array}{l}
e_{x}=\cos (\theta)\left(x_{T}-x\right)+\sin (\theta)\left(y_{T}-y\right) \\
e_{y}=-\sin (\theta)\left(x_{T}-x\right)+\cos (\theta)\left(y_{T}-y\right) \\
e_{\theta}=\theta_{T}-\theta
\end{array}\right.
$$

The error function $e_{R T}$ is added to the canonical error system (2) (cf. Fig. 3). Let us now write $d$ and $\theta_{R T}$ as (cf. Fig. 3):

$$
\begin{aligned}
& d=\sqrt{\left(x_{T}-x\right)^{2}+\left(y_{T}-y\right)^{2}} \\
& \begin{cases}\theta_{R T}=\arctan \left(\left(y_{T}-y\right) /\left(x_{T}-x\right)\right) & \text { if } d>\xi \\
\theta_{R T}=\theta_{T} & \text { if } d \leq \xi\end{cases}
\end{aligned}
$$

where $\xi$ is a small positive value $(\xi \approx 0)$. The error $e_{R T}$ is defined as (cf. Fig. 3):

$$
e_{R T}=\theta_{T}-\theta_{R T}
$$

Furthermore, the velocity set-points of the dynamic target (in global frame $X_{G} Y_{G}$ ) are computed according to the leader velocities and formation shape (cf. Section 3). Finally the pose errors and velocities $\left(e_{x}, e_{y}, e_{\theta}, v_{T}\right)$ are the input of the Control law block (cf. subsection 2.2.3). 


\subsubsection{Obstacle avoidance controller}

Different methods can be found in the literature for obstacle avoidance [14, 24]. One of them is the limit-cycle method, the UGV avoids reactively the obstacle if it tracks accurately limit-cycle trajectories as detailed in [1] (ellipse of influence). The main ideas behind this controller are briefly detailed below:

The differential equations of the elliptic limit-cycles are:

$$
\begin{aligned}
& \dot{x}_{s}=m\left(B y_{s}+0.5 C x_{s}\right)+x_{s}\left(1-A x_{s}^{2}-B y_{s}^{2}-C x_{s} y_{s}\right) \\
& \dot{y}_{s}=-m\left(A x_{s}+0.5 C y_{s}\right)+y_{s}\left(1-A x_{s}^{2}-B y_{s}^{2}-C x_{s} y_{s}\right)
\end{aligned}
$$

with $m= \pm 1$ according to the avoidance direction (clockwise or counter-clockwise). $\left(x_{s}, y_{s}\right)$ corresponds to the position of the UGV according to the center of the ellipse. The variables $A, B$ and $C$ are given by:

$$
\begin{aligned}
& A=\left(\sin (\Omega) / b_{l c}\right)^{2}+\left(\cos (\Omega) / a_{l c}\right)^{2} \\
& B=\left(\cos (\Omega) / b_{l c}\right)^{2}+\left(\sin (\Omega) / a_{l c}\right)^{2} \\
& C=\left(1 / a_{l c}^{2}-1 / b_{l c}^{2}\right) \sin (2 \Omega)
\end{aligned}
$$

where $a_{l c}$ and $b_{l c}$ characterize respectively the major and minor elliptic semi-axes and $c$ gives the ellipse orientation when it is not equal to 0 .

Let us now extend this method, initially proposed for the navigation of a monorobot, to the case of a group of UGVs. In section 3, the formation is defined by longitudinal $h_{i}$ and lateral $l_{i}$ coordinates (15) (cf. Fig 4). At this aim, the dimensions of the ellipse $\left(a_{l c}, b_{l c}\right)$ are increased according to the maximum lateral coordinate of the formation shape $l_{\text {imax }}$, i.e., the dimension of the ellipse to avoid will be $\left(a_{l c}+\right.$ $\left.l_{\text {imax }}, b_{l c}+l_{\text {imax }}\right)$. The advantage of the proposed method is to maintain the shape of the whole formation even when obstacles hinder the formation navigation, instead of each robot avoids locally the obstacles [2].

In our case, the controller can be written as an orientation control. We consider thus $e_{x}=0$ and $e_{y}=0$ in (2) (cf. Fig. 3), i.e, the vehicle position is at each sample time in the desired position. The limit-cycle propriety allows to avoid the obstacles. The desired vehicle orientation is given by the differential equation of the limit-cycle (6) and (7):

$$
\theta_{d}=\arctan \left(\dot{y}_{s} / \dot{x}_{s}\right)
$$

Furthermore, the linear velocities of the UGVs are decreased for safe avoidance when the obstacle avoidance controller is activated, e.g, $v_{T}=v_{\max } / 2$.

\subsubsection{Control law}

The used control law is designed according to Lyapunov stability analysis [23]. The desired vehicle's linear velocity $v$ and its front wheel orientation $\gamma$ that make the errors $\left(e_{x}, e_{y}, e_{\theta}\right)$ converge always to zero can be chosen as: 


$$
\begin{aligned}
& v=v_{T} \cos \left(e_{\theta}\right)+K_{x}\left(K_{d} e_{x}+K_{l} d \sin \left(e_{R T}\right) \sin \left(e_{\theta}\right)+K_{o} \sin \left(e_{\theta}\right) c_{c}\right) \\
& \gamma=\arctan \left(l_{b}\left[r_{c_{T}}^{-1} \cos ^{-1}\left(e_{\theta}\right)+c_{c}\right]\right)
\end{aligned}
$$

where $c_{c}$ is given by:

$$
c_{c}=\frac{d^{2} K_{l} \sin \left(e_{R T}\right) \cos \left(e_{R T}\right)}{r_{c_{T}} K_{o} \sin \left(e_{\theta}\right) \cos \left(e_{\theta}\right)}+K_{\theta} \tan \left(e_{\theta}\right)+\frac{K_{d} e_{y}-K_{l} d \sin \left(e_{R T}\right) \cos \left(e_{\theta}\right)}{K_{o} \cos \left(e_{\theta}\right)}+\frac{K_{R T} \sin ^{2}\left(e_{R T}\right)}{\sin \left(e_{\theta}\right) \cos \left(e_{\theta}\right)}
$$

$\mathbf{K}=\left(K_{d}, K_{l}, K_{o}, K_{x}, K_{R T}, K_{\theta}\right)$ is a vector of positive constants which must be defined by the designer. $K_{d}, K_{l}$ and $K_{o}$ are respectively related to the desired convergence of the distance, lateral and angular errors w.r.t. the assigned target. $K_{x}, K_{R T}$ and $k_{\theta}$ are related to the maximum linear and angular velocities (more details are given in [23]).

\section{Navigation in formation}

We consider a group of $N$ UGVs with the objective of reaching and keeping their assigned configuration according to the desired formation and leader configuration $[8,25]$. The proposed strategy consists on controlling each UGV (follower) to track its assigned virtual dynamic target (cf. subsection 3.1 and Fig. 4). The strategy of formation reconfiguration (cf. subsection 3.2) is based on a suitable smooth switch of these virtual dynamic targets while considering the inter-vehicle collisions.

\subsection{Leader-follower approach}

Leader-follower approach allows to maintain a rigid geometric shape (e.g. a triangle in Fig. 4). The formation is defined in this case w.r.t. the Cartesian frame (local frame of the leader) (cf. Fig 4). The proposed formation, based on Leader-follower approach, is defined by:

- A leader $\left(\mathrm{UGV}_{L}\right.$ in Fig. 4$)$; its pose $\left(x_{L}, y_{L}, \theta_{L}\right)$ and its linear velocity $v_{L}$ determine the dynamic of the formation (cf. Fig. 4).

- The formation structure is defined with as much nodes as necessary to obtain the desired formation shape. Each node $i$ is a virtual dynamic target $\left(T_{d_{i}}\right)$. The formation is defined as $\mathbf{F}=\left\{\mathbf{f}_{i}, i=1 \cdots N\right\}$, where $\mathbf{f}_{i}$ are the coordinates $\left(h_{i}, l_{i}\right)^{T}$ of the dynamic target $T_{d_{i}}$ w.r.t. the leader local reference frame (cf. Fig. 4).

The position and orientation of each node (virtual target) are computed from the leader configuration. The leader position determines the nodes positions according to the formation shape. The instantaneous center of curvature $I_{c c_{L}}$ of the formation is determined by the leader according to its movements (cf. Fig. 4). $I_{c c_{L}}$ allows to compute the desired orientation of the nodes according to the formation shape (cf. 
Fig. 4). The leader turns around $I_{c c_{L}}$ (positioned perpendicularly to its rear wheel), then the other target set-points $T_{d_{i}}$ must also turn around $I_{c c_{L}}$ to maintain the rigid formation. Thus, the target velocity $v_{T_{i}}$ must be tangent to the circle which has $I_{c c_{L}}$ as center and the distance between $T_{d_{i}}$ and $I_{c c_{L}}$ as radius $r_{c_{T_{i}}}$.

The idea behind this strategy is to eliminate the dependency of each UGV to a global reference frame. A straightforward transformation can be applied to obtain the set-point w.r.t. a local reference frame attached to the leader. The polar coordinates $\left(r_{i}, \Phi_{i}\right)$ can also be used by applying a straightforward transformation. An important advantage of the used Leader-follower approach is that it does not depend here on any reference trajectory and the formation is fully defined by the instantaneous dynamic of the leader. An important advantage of the proposed formation definition based on Leader-follower approach is that it takes, in addition to the target positions $\left(x_{T}, y_{T}\right)$, the heading $\theta_{T}$ of the virtual targets, which allows to have even more accurate formation navigation (cf. section 4). Furthermore, the proposed approach is more reactive in the sense that it takes at each sample time only the current configuration and velocity of the Leader, instead of using the trajectory of the Leader as a reference for the formation $[5,20]$.

One important consideration to take into account to achieve the presented navigation of formation strategy, is that the followers have to know, as accurately as possible, the leader state (pose and velocity). As mentioned before, we assume that the leader sends its state by stable Wi-Fi communication without latency. However, cameras and/or LIDAR sensors embedded in each follower, can be used to estimate the leader state $[8,11]$.

In the sequel, $\mathbf{f}_{i}$ is given in Global Cartesian frame to homogenize the notation of the equations. The pose of the virtual target $T_{d_{i}}$ w.r.t the leader pose in the Global reference frame can be written as (cf. Fig. 4):

$$
\left\{\begin{array}{l}
x_{T_{i}}=x_{L}+h_{i} \cos \left(\theta_{L}\right)-l_{i} \sin \left(\theta_{L}\right) \\
y_{T_{i}}=y_{L}+h_{i} \sin \left(\theta_{L}\right)+l_{i} \cos \left(\theta_{L}\right) \\
\theta_{T_{i}}=\theta_{L}+\beta_{i}
\end{array}\right.
$$

where $\left(x_{L}, y_{L}, \theta_{L}\right)$ is the current pose of the leader and $\beta_{i}$ is the $T_{d_{i}}$ orientation w.r.t. the leader pose. It is given by:

Fig. 4 Formation definition in mobile Cartesian frame linked to the Leader.

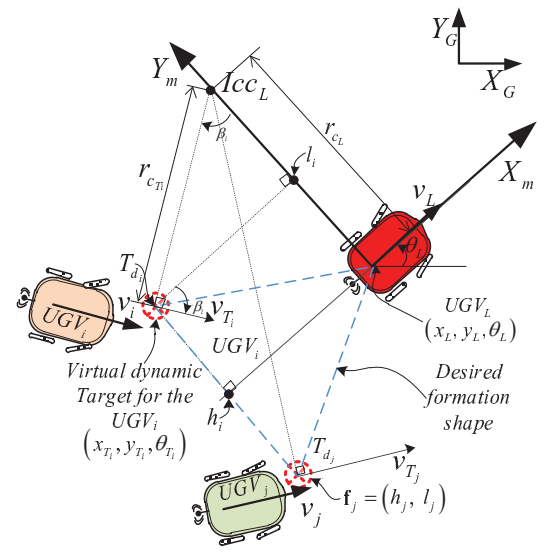




$$
\beta_{i}=\arctan \left(h_{i} /\left(r_{c_{L}}-l_{i}\right)\right)
$$

where $r_{c_{L}}$ is the radius of curvature of the leader. Differentiating (15), the velocities of each $T_{d_{i}}$ are given thus by:

$$
\begin{aligned}
v_{T_{i}} & =\sqrt{\left(v_{L}-l_{i} \omega_{L}\right)^{2}+\left(h_{i} \omega_{L}\right)^{2}} \\
\omega_{T_{i}} & =\omega_{L}+\dot{\beta}_{i}
\end{aligned}
$$

where $v_{L}$ and $\omega_{L}$ are respectively the linear and angular velocities of the leader, $\dot{\beta}_{i}$ is computed as:

$$
\dot{\beta}_{i}=-h_{i} \dot{r}_{c_{L}} /\left(\left(r_{c_{L}}-l_{i}\right)^{2}+\left(h_{i}\right)^{2}\right)
$$

One can note from (19) that when $\dot{\beta}_{i}$ is equal to zero, the formation has a constant radius of curvature $r_{c_{L}}$ and the angular velocities of the virtual targets are equal to the angular velocity of the leader $\left(\omega_{T_{i}}=\omega_{L}\right)(18)$.

\subsection{Proposed strategy for formation reconfiguration}

Different methods dealing with formation reconfiguration for a group of UGV have been proposed in the literature [4, 5, 8]. Many methods exploit Model Predictive Control (MPC) based on time horizon and optimization of a cost function [4]. These methods are generally time consuming due to predictive computation w.r.t. a time horizon. Moreover, they were applied to small unicycle robots and they are based on predefined trajectories computed along a time horizon. This subsection proposes a new Strategy for Formation Reconfiguration (SFR) based on suitable smooth switches between different virtual target configurations (cf. subsection 3.2.1). This strategy allows to obtain a fully reactive architecture in the sense that the UGV followers track the instantaneous state (pose and velocity) of its virtual targets (thus, without any use of a reference trajectory or a trajectory planning process). Additionally to the reconfiguration process, one should manage potential collisions between UGVs and allocation of virtual targets to UGVs (cf. Subsection 3.2.2).

Different algorithms optimizing target assignment can be easily integrated in our multi-block control architecture (cf. Fig. 2) (refer to [2, 25]). Nonetheless, this paper is focused on the control strategy for formation reconfiguration. Therefore, the allocation of virtual targets to UGVs is achieved using elementary rules when a formation reconfiguration is required (cf. section 4). These rules assign a label $H_{i}$ of the virtual target $T_{d_{i}}$ to the $U G V_{i}$ at the beginning of the experiments. This label is kept by each UGV along of the reconfiguration process (cf. Fig. 5).

\subsubsection{Reconfiguration method}

A typical example of application of formation reconfiguration is when the formation detects a narrow tight corridor, therefore the formation have to adapt to the corridor 
dimension to continue the navigation. The proposed strategy for formation reconfiguration is based on suitable smooth switching of the virtual target configurations. The new virtual targets defined on the new formation shape must be ahead to the UGVs to guarantee the stability of the overall system (the vehicle must not go back to reach the new virtual target). If this condition is not satisfied then the former formation will be adapted by increasing smoothly and contentiously the longitudinal coordinates $h_{i}$ until that all UGVs will be positioned in the right configuration (21). The error between the coordinates of the former and the new formation $\mathbf{e}_{f_{i}}\left(e_{h_{i}}, e_{l_{i}}\right)$ is defined as:

$$
\mathbf{e}_{f_{i}}=\mathbf{f}_{i}^{n}-\mathbf{f}_{i}^{f}
$$

where $\mathbf{f}_{i}^{f}\left(h_{i}^{f}, l_{i}^{f}\right)$ and $\mathbf{f}_{i}^{n}\left(h_{i}^{n}, l_{i}^{n}\right)$ are respectively the coordinates of the former formation and new desired formation (cf. Fig. 4 and 5).

The reconfiguration process between the different formation shapes is given by:

$$
\mathbf{f}_{i}= \begin{cases}h_{i}=h_{i}^{n}-e_{h_{i}} e^{-k_{r}\left(t-t_{r}\right)}, & l_{i}=l_{i}^{n} ; \text { if } e_{h_{i}}<0 \\ h_{i}=h_{i}^{n}, & l_{i}=l_{i}^{n} ; \text { if } e_{h_{i}} \geq 0\end{cases}
$$

where $\mathbf{f}_{i}\left(h_{i}, l_{i}\right)$ are the coordinates of the current virtual target $T_{d_{i}}$ to be tracked by the follower $\mathrm{UGV}_{i} . e_{h_{i}}$ is the longitudinal coordinate of $\mathbf{e}_{f_{i}}$ that allows to detect if the virtual target is ahead to its respective follower $\left(e_{h_{i}} \geq 0\right)$. The adaptation function when $e_{h_{i}}<0$ (virtual target behind to follower ${ }_{i}$ ) is set as proportional to the error between formation shapes, where $k_{r}$ is a real positive constant designed according to the dynamic of the leader and $t_{r}>0$ is the initial time for the reconfiguration process. An accurate analysis of this adaptive reconfiguration function will be developed in future works.

\subsubsection{Collision between UGV}

Collision between UGVs can occur during the reconfiguration phase of the group of UGVs. To address this collision risk, we use a penalty function acting on the linear velocity of the UGVs. Moreover, this reduced velocity of UGVs allows to obtain a smooth and less oscillating vehicles' movements (cf. section 4). Each UGV is enclosed by two circle $C_{i n t}$ and $C_{\text {ext }}$ with respectively radius $R_{i n t}$ and $R_{\text {ext }}\left(R_{i n t}<\right.$ $\left.R_{\text {ext }}\right)$. The collision occurs when the distance $d_{i j}$ between $U G V_{i}$ and $U G V_{j}$ are less than $R_{\text {int }}$. Hence, the penalty function $\psi_{i}^{j}$ for the $U G V_{i}$ w.r.t. the $U G V_{j}$ is defined as:

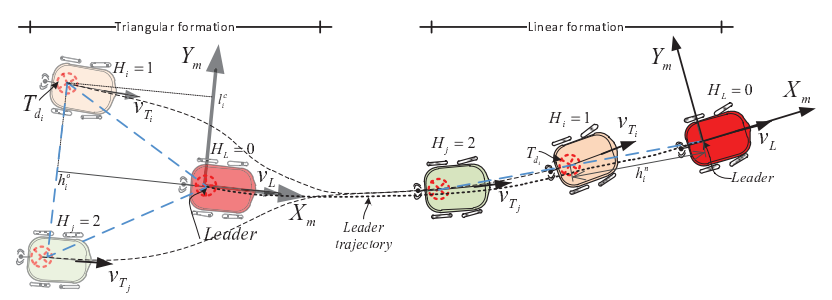

Fig. 5 Formation reconfiguration between, for instance, triangular and linear formation shapes.

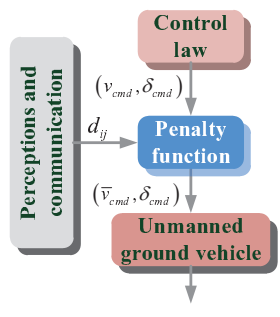

Fig. 6 Integration of the penalty function in the proposed architecture. 


$$
\psi_{i}^{j}= \begin{cases}1 & \text { if } d_{i j} \geq R_{e x t} \\ \left(d_{i j}-R_{\text {int }_{i}}\right) /\left(R_{\text {ext }}-R_{\text {int }}\right) & \text { if } R_{\text {int }}<d_{i j}<R_{\text {ext }} \\ 0 & \text { if } d_{i j} \leq R_{\text {int }}\end{cases}
$$

The modified linear velocity of the $U G V_{i}$ is then given by:

$$
\bar{v}_{j}=v_{j} \psi_{i}^{j}
$$

Using the definition of $R_{\text {int }}$ (where $R_{\text {int }} \neq R_{\text {int }}$ ), it is guaranteed that two UGVs do not stop simultaneously. Indeed, if the UGVs have the same $R_{\text {int }_{i}}$ we can observe local minima in certain configurations, in fact, when $d_{i j}<R_{\text {int }_{i}}$ then $\psi_{i}^{j}=\psi_{j}^{i}=0$ and the robots are stopped at the same time.v $R_{e x t}$ is designed according to communication constraints (latency) and localization errors (GPS). This penalty function can be straightforward integrated to our control architecture (cf. Fig. 2) by adding a block after the output of the Control law block (cf. Fig. 6).

\section{Simulations}

This section shows the navigation of a group of $N=3$ UGVs in a cluttered environment using the proposed control architecture. The reconfiguration strategy (SFR) between the formation shapes is also analyzed. All simulations were made in MATLAB ${ }^{\circledR}$ software. The physical parameters of the used UGV are based on the urban vehicle VIPALAB from Apojee company [13]. The UGV constraints are minimum velocity $v_{\min }=0.1 \mathrm{~m} / \mathrm{s}$, maximum velocity $1.5 \mathrm{~m} / \mathrm{s}$, maximum steering angle $\gamma_{\max }= \pm 30^{\circ}$ and maximum acceleration $1.0 \mathrm{~m} / \mathrm{s}^{2}$. We consider that the sample time is $0.01 s$. Each UGV has a range sensor (LIDAR) with a maximum detected range equal to $D_{\max }=10 \mathrm{~m}$ and a stable communication network.

The controller parameters are set to $\mathbf{K}=(1,2.2,8,0.1,0.01,0.6)$ (cf. subsection 2.2.3). These parameters were chosen to obtain a safe and smooth trajectory, fast response and velocity value within the limits of the vehicle capacities. The radius for non-collision between UGVs are selected as $R_{\text {int }_{L}}=1.8 \mathrm{~m}, R_{\text {int }}=2.2 \mathrm{~m}$, $R_{\text {int }_{2}}=2.0 \mathrm{~m}$ and $R_{\text {ext }}=2.7 \mathrm{~m}$. For each simulation the vehicles start at the same configuration and must reach the same final configuration. The initial positions of the vehicles have an offset $(\Delta x, \Delta y)=(1,0.5) m$ from the initial position of their assigned virtual targets.

The simulations given in Fig. 7 to 11 are selected from several conclusive simulations because they make a focus on the proposed reconfiguration method between the two formation shape (triangular and linear shapes) while navigating in a cluttered environment (this simulation can be found online ${ }^{1}$ ). We consider that the initial formation coordinates are defined by $\mathbf{F}=\left(\mathbf{f}_{1}, \mathbf{f}_{2}\right)$, with $\mathbf{f}_{1}=(-4,-2)^{T} m$ and $\mathbf{f}_{2}=(-4,2)^{T} m$ (triangular shape). Therefore, the group of UGVs must keep the formation while moving in a cluttered environment. A static target is defined in the

\footnotetext{
${ }^{1}$ http://maccs.univ-bpclermont.fr/uploads/Profiles/VilcaJM/FormationReconfiguration.mp4
} 
environment, the leader (and thus the formation) must go toward it while avoiding the hinder obstacle. The new targeted formation is defined as straight line with the following coordinates $\mathbf{F}^{n}=\left(\mathbf{f}_{1}^{n}, \mathbf{f}_{2}^{n}\right)$, with $\mathbf{f}_{1}^{n}=(-6,0)^{T} m$ and $\mathbf{f}_{2}^{n}=(-3,0)^{T} m$.

At the beginning of the simulation (cf. Fig. 9), the navigation of the group of UGVs is in triangular formation $\mathbf{F}$. When the leader detects an obstacle with adequate range to allow the formation reconfiguration, then the leader avoids the obstacle using the limit-cycle method (limit-cycle is increased by $R_{f}=2 \mathrm{~m}$ to allow a safe navigation (cf. subsection 2.2.2) and sends the new desired formation $\mathbf{F}^{n}$ to the other UGVs (followers) to modify the configuration of the formation. The formation returns to triangular shape $\mathbf{F}$, when the leader does not detect obstacles that can hinder the other UGVs movement and the last follower left behind the avoided obstacle. The adaptation phase allows to have the virtual target always ahead to the followers to obtain a suitable adaptive formation reconfiguration (cf. Fig. 7 and 11).

Figure 7 shows the values of errors $d$ and $e_{\theta}$ between each UGV and its virtual target. At first reconfiguration, it can be observed that the follower 1 wait until its assigned virtual target is ahead (cf. Subection 3.2.1). Moreover, it is noted some small peaks that are related to the fast dynamic change of the leader (the dynamic of the formation increased and the saturation occurs in the followers when the leader curvature increased). Fig. 8 shows the distance between each UGV of the formation. This last figure shows clearly non-collision between the vehicles in the formation, i.e., $d_{i j}>R_{i n t_{12}}$ (cf. Subsection 3.2.2). The figures show some peaks which are related to the adaptation and reconfiguration phase between formations.

Figures 9 and 10 show respectively the trajectories and velocities of the UGVs. It can be noted that the vehicles trajectories are smooth along the navigation and there is not neither collisions with the obstacles nor inter-vehicle collisions. The reconfiguration strategy was designed to reduce the peaks of the control commands of each UGV when the transition between the formation occurs (cf. Fig. 10). The proposed control architecture allows thus to adapt the formation according to the environment context. Fig. 11 shows the evolution of the formation coordinates $\left(h_{i}, l_{i}\right)$ (virtual target positions). It can be observed that adaptation phase of $h_{i}$ when the follower is always ahead of its new assigned virtual target (21) which attest on the efficiency of the strategy for formation reconfiguration.

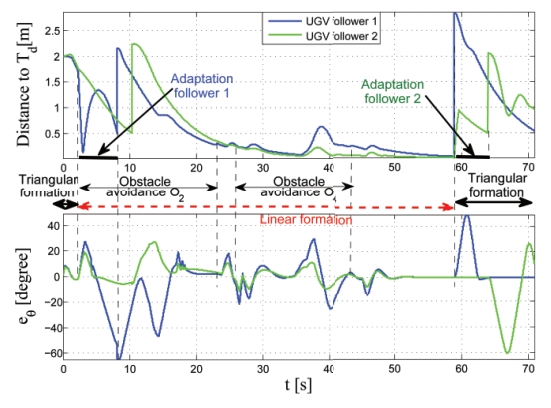

Fig. 7 Distance and orientation errors of the UGVs w.r.t. their virtual targets.

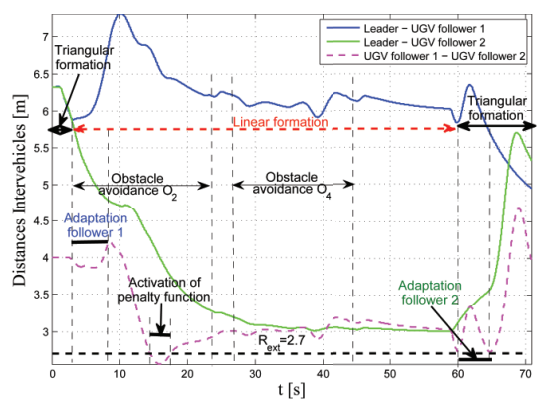

Fig. 8 Distance among the UGVs. 


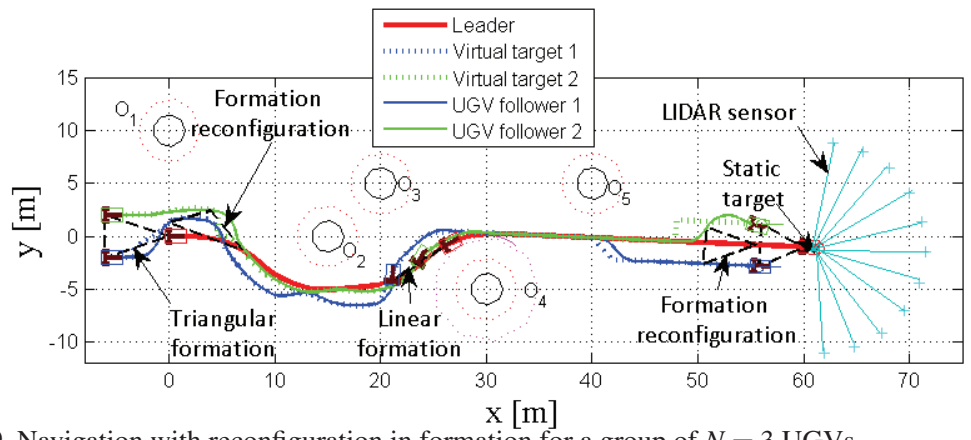

Fig. 9 Navigation with reconfiguration in formation for a group of $N=3 \mathrm{UGVs}$.

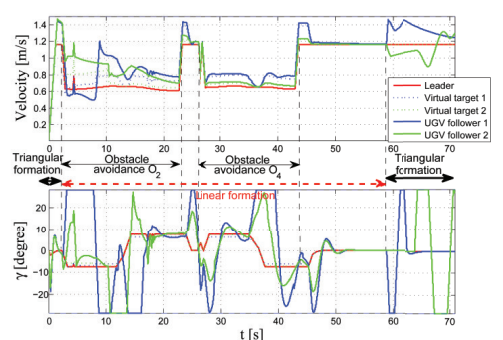

Fig. 10 Velocities commands of the UGVs.

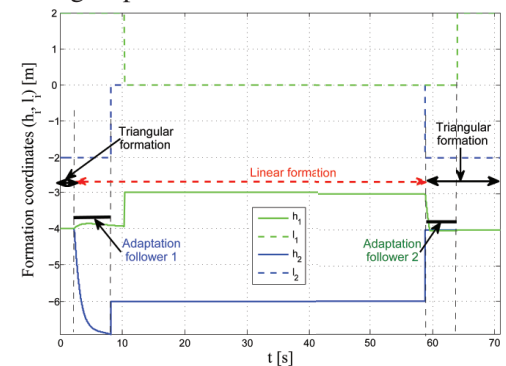

Fig. 11 Progress of the set-point definition $\mathbf{f}_{i}$ according to the proposed SFR.

\section{Conclusions and prospects}

This paper presented an overall control architecture to cope with the navigation in formation of a group of UGVs in cluttered environment. A single Control law embedded in each UGV is used in the proposed architecture which allows the simplification of the overall control strategy for the navigation in formation. The obstacle avoidance based on the limit-cycle trajectories allows to keep the desired formation shape during the navigation even in cluttered environments. In the proposed formation definition based on Leader-follower approach, the leader reference path is not taken into account, only its current pose and dynamic has to be known by the followers. It allows thus more accurate and flexible formation navigation. A fully reactive reconfiguration strategy between the UGVs based on suitable smooth switching of the virtual target configurations was also proposed. This strategy avoids the use of predefined trajectories and it can be applied for different situations when the formation has to be modified according to the environment context (dynamic, cluttered, etc.). Furthermore, this strategy takes into account the probable collisions between vehicles as well as the vehicle constraints to ensure safe navigation to reach the new desired formation. Different accurate simulations using a tricycle vehicles show the efficiency and the flexibility of the proposed strategy for multi-robot navigation.

In future works, formation reconfiguration strategy even in uncertain environments (for instance, w.r.t. the vehicle's perception/localization) will be addressed. 


\section{References}

1. Adouane, L., Benzerrouk, A., Martinet, P.: Mobile robot navigation in cluttered environment using reactive elliptic trajectories. In: 18th IFAC World Congress (2011)

2. Benzerrouk, A., Adouane, L., Martinet, P.: Altruistic distributed target allocation for stable navigation in formation of multi-robot system. In: 10th International IFAC Symposium on Robot Control (SYROCO’12). Dubrovnik - Croatia (2012)

3. Chaimowicz, L., Kumar, R.V., Campos, M.F.M.: A mechanism for dynamic coordination of multiple robots. Autonomous Robots 17, 7 - 21 (2004)

4. Chao, Z., Zhou, S.L., Ming, L., Zhang, W.G.: UAV formation flight based on nonlinear model predictive control. Mathematical Problems in Engineering 2012, 1-15 (2012)

5. Chen, X., Li, Y.: Smooth formation navigation of multiple mobile robots for avoiding moving obstacles. International Journal of Control, Automation 4(4), 466 - 479 (2006)

6. Clark, J., Fierro, R.: Mobile robotic sensors for perimeter detection and tracking. \{ISA\} Transactions 46(1), 3 - 13 (2007)

7. Consolini, L., Morbidi, F., Prattichizzo, D., Tosques, M.: Leader-follower formation control of nonholonomic mobile robots with input constraints. Automatica 44(5), 1343 - 1349 (2008)

8. Das, A., Fierro, R., Kumar, V., Ostrowski, J., Spletzer, J., Taylor, C.: A vision-based formation control framework. IEEE Transaction on Robotics and Automation 18(5), 813-825 (2002)

9. Dasgupta, P., Whipple, T., Cheng, K.: Effects of multi-robot team formations on distributed area coverage. International Journal of Swarm Intelligent Research 2(1), 44-69 (2011)

10. Desai, J., Ostrowski, J., Kumar, V.: Modeling and control of formations of nonholonomic mobile robots. IEEE Transaction on Robotics and Automation 17(6), 905 - 908 (2001)

11. El-Zaher, M., Contet, J.M., Gechter, F., Koukam, A.: Echelon platoon organisation: a distributed approach based on 2-spring virtual links. In: 15th International Conference on Artificial Intelligence: Methodology, Systems, Applications (AIMSA). Germany (2012)

12. Ghommam, J., Mehrjerdi, H., Saad, M., Mnif, F.: Formation path following control of unicycle-type mobile robots. Robotics and Autonomous Systems 58(5), 727 - 736 (2010)

13. The Institut Pascal Data Sets: (2013). URL http://ipds.univ-bpclermont.fr.

14. Khatib, O.: Real-time obstacle avoidance for manipulators and mobile robots. The International Journal of Robotics Research 5, pp.90-99 (1986)

15. Levinson, J., Thrun, S.: Robust vehicle localization in urban environments using probabilistic maps. In: IEEE International Conference on Robotics and Automation. Alaska, USA (2010)

16. Lozenguez, G., Adouane, L., Beynier, A., Mouaddib, A., Martinet, P.: Map partitioning to approximate an exploration strategy in mobile robotics. MAGS - Multiagent and Grid Systems (2012)

17. Luca, A.D., Oriolo, G., Samson, C.: Feedback control of a nonholonomic car-like robot. In: J.P. Laumond (ed.) Robot Motion Planning and Control, pp. 171-253. Springer-Verlag (1998)

18. Mesbahi, M., Hadaegh, F.: Formation flying control of multiple spacecraft via graphs, matrix inequalities, and switching. In: Control Applications, 1999. Proceedings of the 1999 IEEE International Conference on, vol. 2, pp. 1211-1216 vol. 2 (1999)

19. Miner, D.: Swarm robotics algorithms: A survey (2007)

20. Shames, I., Deghat, M., Anderson, B.: Safe formation control with obstacle avoidance. In: IFAC World Congress. Milan - Italy (2011)

21. Sinha, A., Ghose, D.: Generalization of linear cyclic pursuit with application to rendezvous of multiple autonomous agents. IEEE Transactions on Automatic Control 51, 1819-1824 (2006)

22. Tang, H., Song, A., Zhang, X.: Hybrid behavior coordination mechanism for navigation of reconnaissance robot. In: International Conference on Intelligent Robots and Systems (2006)

23. Vilca, J., Adouane, L., Mezouar, Y., Lébraly, P.: An overall control strategy based on target reaching for the navigation of an urban electric vehicle. In: IEEE/RSJ International Conference on Intelligent Robots and Systems (IROS'13). Tokyo - Japan (2013)

24. Zapata, R., Cacitti, A., Lepinay, P.: Dvz-based collision avoidance control of non-holonomic mobile manipulators. JESA, European Journal of Automated Systems 38(5), 559-588 (2004)

25. Ze-su, C., Jie, Z., Jian, C.: Formation control and obstacle avoidance for multiple robots subject to wheel-slip. International Journal of Advanced Robotic Systems 9, 1-15 (2012) 